\title{
Subsistema de operaciones en empresas de servicios: Una reflexion teórica
}

\author{
Diz Comesaña, María Eva*
}

\section{Resumen}

Este trabajo tiene como propósito realizar una reflexión teórica sobre los elementos que participan en la prestación del servicio y cómo las modificaciones provocadas en los mismos por la incorporación de procesos automatizados podrían mejorar los resultados del subsistema de operaciones. Para ello se ha realizado una revisión teórica de la literatura más importante. Los resultados han permitido constatar que la automatización se manifiesta como una posible vía dentro del sector terciario para la obtención de ventajas competitivas, posibilitando incluso la consecución de cotas aceptables de productividad y flexibilidad de forma simultánea, prioridades competitivas tradicionalmente antagónicas. Se concluye que si bien la automatización puede permitir conseguir los objetivos, no siempre es la opción más favorable para la empresa, puesto que es necesario adaptarse a la situación de cada organización.

Palabras clave: Servicios, Automatización, Subsistema de Operaciones.

\section{Operational Subsystems in Service Enterprises: A Theoretical Reflection}

\section{Abstract}

The purpose of this paper is to undertake a theoretical reflection as to the elements that participate in the offer of services and in what manner the modifications provoked in the same due to the incorporations of automated processes could improve the results of the operational sub-system. In order to do this a theoretical review of literature on this theme was undertaken. The results allowed us to determine that automation is indeed a possible manner of obtaining competitive advantages in the third sector, permitting the achievement of acceptable levels of productivity and flexibility simultane-

Recibido: 04-02-17. Aceptado: 05-10-10

Diplomada en Ciencias Empresariales, Licenciada en Ciencias Económicas y Empresariales, Suficiencia como Investigadora, Doctora en Administración y Dirección de Empresas. Profesora de la Universidad de Vigo desde el año 1994. E-mail: evadiz@uvigo.es 
ously, competitive priorities which were traditionally antagonistic. The conclusion is that when automation could facilitate the achievement of objectives, it is not always the most favourable option for a company since it is necessary to adapt the situation in each particular case and organization.

Key words: Services, automation, operational subsystem.

\section{Introducción}

Tradicionalmente se ha tratado de reflejar a grandes rasgos el proceso realizado por el subsistema de operaciones en la producción de bienes o servicios, independientemente de la empresa o sector al que pertenezca, tratando de recoger los elementos básicos comunes en estos procesos.

Tal como indican Adam y Ebert (1991: 5), para la obtención del producto es necesario un proceso de conversión que tratará de transformar los insumos en bienes y/o servicios; por otro lado, también resulta interesante mantener un proceso de retroalimentación de información, de tal forma que los resultados se adapten a lo deseado. Una vez obtenidos los bienes y servicios, éstos pasarán a la venta (en el caso de los servicios lo normal es que la producción tenga lugar en el momento de la venta), donde se transforman en efectivo, este dinero permitirá adquirir más recursos para mantener en funcionamiento el proceso de conversión, y así sucesivamente. La meta general para este tipo de sistemas consiste en generar cierto valor añadido, de tal forma que los consumidores valoren los productos en un importe superior a la suma de los recursos utilizados. Para el consumidor, los productos resultantes del proceso de conversión deben ofrecer algún tipo de utilidad.
El proceso de conversión es distinto dependiendo del tipo de empresa, pero tiene lugar en todas ellas aunque se trate del sector servicios. Podría decirse que la transformación de insumos en bienes y/o servicios varía de forma importante dependiendo de las tecnologías utilizadas, entendiéndose como tales no solamente los tipos de actividades de transformación que concurren, sino que habría que incluir la complejidad científica de la planta, de la maquinaria, de las habilidades y de los bienes y/o servicios que tienen lugar en el proceso.

Las fluctuaciones aleatorias son influencias no controladas que pueden originar diferencias entre el producto real y el que había sido planeado. Estas fluctuaciones tanto pueden ser externas como internas, y sus efectos pueden ser muy importantes, de hecho, en los procesos de producción constituyen la regla más que la excepción. Por tanto, una de las principales tareas de la empresa, consistirá en la disminución de estas variaciones en la fuente y/o su correcta gestión en el punto de impacto (Harvey et al., 1997).

Por otro lado, la función de retroalimentación es otro de los factores a tener en cuenta. Esta función es la encargada de proporcionar enlaces de información. Si no tuviese lugar la retroalimentación de información, la dirección no podría controlar las operaciones porque desconocería los resultados de sus decisiones. 
Cada servicio posee un valor, tanto desde el punto de vista del que lo ofrece como del consumidor, su cliente. Pero este valor solamente se hace efectivo bajo dos condiciones: por un lado, que los recursos materiales y humanos pertenecientes a la empresa de servicios estén disponibles, y por otro, que el cliente sienta la necesidad y acudiendo a la empresa de servicios la satisfaga (Eiglier y Langeard, 1993:3). Por tanto, el valor del servicio existe cuando tiene lugar la producción del mismo".

Por ello se ha creído conveniente estudiar de forma genérica el subsistema de operaciones de las empresas de servicios, tratando de concretar aquellos elementos que participan en la prestación del mismo -personal en contacto, soporte físico, sistema de organización interna, cliente, otros clientes y servicio-. Una vez hecho esto, se ha considerado la incorporación de procesos automatizados, los cuales introducen modificaciones tanto en los elementos que participan en la prestación del servicio como en las relaciones que éstos mantienen entre sí.

Estos cambios podrían producir mejoras en los resultados del subsistema de operaciones. En este sentido y buscando una mayor concreción, se ha creído oportuno tomar aquellas variables consideradas tradicionalmente como ob- jetivos de dicho subsistema -coste, tiempo, calidad, flexibilidad y servicio al cliente- y estudiar su evolución.

\section{El subsistema de operaciones}

Analizado el Subsistema de Operaciones desde una perspectiva genérica y centrando ahora la atención exclusivamente en las empresas de servicios, se ha tratado de reflejar los puntos en común en la producción de los mismos, para ello se han tenido en cuenta los distintos recursos generadores del servicio. En la organización productora del servicio hay varias funciones o departamentos implicados, sin embargo desde la perspectiva del cliente son percibidos como un sistema integral -o al menos esto debiera ocurrir-. El cliente es entendido también como un recurso de la producción del servicio, no obstante, en ocasiones las empresas de servicios lo consideran como consumidor olvidando la faceta de coproductor (Tansik, 1988:1). Esto es debido al papel atribuido a los clientes en los enfoques clásicos que siguen la orientación hacia la producción, es decir, que utilizan las mismas metodologías empleadas por las empresas manufactureras (Chase, 1988). Sin embargo, en el sector terciario los clientes actúan en conexión con la

1 Pierre Eiglier y Eric Langeard en vez de utilizar el término "producción" de servicios, crean un nuevo vocablo "servucción" ( servuction), que consideran más apropiado, puesto que el primero -según su opinión- se refiere a la producción de productos, entendidos éstos como bienes tangibles. Esta postura es aceptada por otros autores que siguen utilizando la misma terminología. Sin embargo, en este trabajo se ha optado por el vocablo producción tanto para hacer referencia a la fabricación de bienes tangibles como a la de servicios, sin menospreciar por ello la postura anterior. 
empresa, es decir, interactúan con las demás partes del subsistema de producción (Grönroos, 1994: 201).

No obstante, el cliente externo solamente entra en contacto con una parte del subsistema, ya que existe lo que Shostack $(1984,1987)$ ha dado en llamar línea de visibilidad, que divide aquellas partes del proceso que el cliente percibe con claridad de otras que influyen de forma indirecta sobre la calidad del servicio percibida, es decir, que no son visibles por el cliente, pero no por ello deben ser desatendidas.

Respecto a este elemento -clientees importante conocer sus expectativas, influenciadas por sus necesidades y deseos, sus experiencias previas, la imagen de la empresa a nivel global y local, los instrumentos de comunicación o promoción en marketing, la comunicación boca a boca, la ausencia de comunicación, etc.

Por otro lado, se debe tener en cuenta la misión de la empresa así como los correspondientes conceptos del servicio, que deben actuar como guías para planificar y gestionar el sistema de producción; y también la cultura de la empresa -normas y valores compartidos que manifiestan lo que se piensa y valora dentro de la organización-, la cual siempre está presente aunque su impacto sobre los empleados puede ser mayor o menor.

\subsection{Encuentro del Servicio}

Una vez analizado el subsistema de operaciones de las empresas de servicios, puede concluirse que la mayoría de los servicios son el resultado de procesos que se realizan en su mayor parte, sino en su totalidad, en contacto directo entre el cliente y los representantes de la empresa (personal en contacto), este momento es lo que Normann (1990: 25) a dado en llamar "la hora de la verdad". Este encuentro (Service Encounter) tiene lugar, generalmente, bajo el marco de la empresa prestataria del servicio. Intervienen, por tanto, tres elementos -cliente, personal en contacto y organización- que interactúan entre sí (Bateson, 1985a: 76; Solomon et al.,1985), teniendo cada uno sus prioridades y objetivos, entre los que pueden surgir diversos conflictos en ocasiones difíciles de reconciliar².

La organización, establece el entorno físico y cultural del encuentro del servicio. El personal actúa con cierta autonomía ya que la cultura empresarial le proporciona una serie de valores que, en cierto modo, controlan su comportamiento, lo que desencadena una menor necesidad de supervisión directa. No obstante, la cultura por sí sola no asegura el éxito de la autonomía de los empleados a la hora de tomar decisiones. Es importante que el personal esté informado, motivado, formado y que resulte competente

2 Parasuraman (1996) analiza las relaciones que la tecnología mantiene con estos elementos. Agnihothri et al (2002) estudian como la tecnología influye en las relaciones que estos elementos mantienen entre sí. 
para el desempeño de su función ${ }^{3}$. Entre estos tres elementos se establecen una serie de relaciones que se materializan del siguiente modo:

- Interacción entre la organización de servicios y el personal en contacto. La organización suele establecer ciertas reglas y procedimientos para limitar la autonomía del personal en contacto a la hora de prestar el servicio y así disponer de un mayor control, lo que puede provocar un enfrentamiento entre la eficiencia a obtener por parte de la empresa y la autonomía que reclama el personal en contacto. Aunque, tal como se ha indicado, una correcta cultura empresarial, junto con la información, motivación, formación y competencia adecuada para el personal en contacto, podría hacer que la autonomía desemboque en una mayor eficiencia y eficacia. No obstante, con la incorporación de las nuevas tecnologías y la reducción o desaparición del personal en contacto, la empresa suele obtener un nivel de eficiencia más elevado.

- Interacción entre la organización de servicios y el cliente. Las reglas y procedimientos propuestos por la organización pueden suponer una pérdida de adaptación del servicio al cliente, lo que podría desembocar en un cliente insatisfecho. De ahí el enfrentamiento entre la eficiencia a obtener por parte de la empresa y la satisfac- ción del cliente, aunque esta última podría ser considerada como una mayor eficacia para la empresa. La eficiencia será mayor cuando las expectativas del cliente y el papel que desempeña en la prestación del servicio sean conocidos.

- Interacción entre el persoÿal en contacto y el cliente. Esta interacción suele generar cierto grado de control percibido y buscado por ambas partes. Por un lado, el personal en contacto tratará de controlar el comportamiento del cliente para tener así menos complicaciones. $\mathrm{Y}$, por otro lado, el cliente también intentará disponer de cierto control en la prestación del servicio para obtener el máximo partido.

Estas relaciones entre los tres elementos tratados deben alcanzar cierto equilibrio para que el encuentro resulte satisfactorio para todas las partes, satisfacción que debería de equilibrar la necesidad de control por parte de los participantes. Cuando uno de los elementos asume la mayoría del control del proceso surgen ciertos desequilibrios tales como (Bateson, 1985a:76):

- Encuentro dominado por la organización de servicios. En aquellas empresas que siguen básicamente una estrategia competitiva de liderazgo en costes, al tratar de conseguir altos niveles de eficiencia se estandariza la prestación del servicio, lo que, por

3 Las Tecnologías de la Información pueden desempeñar un importante papel para que los trabajadores dispongan de la información suficiente por parte de la organización (Schlessinger y Heskett, 1991: 72), así como soporte de la relaciones que mantienen con los consumidores (Mulligan y Gordon, 2002). 
una parte, acotará el grado de autonomía del personal en contacto y, por otra, enfrentará al cliente con una serie de alternativas de servicio limitadas, lo cual implica un grado de personalización menor.

- Encuentro dominado por el personal en contacto. El personal en contacto, generalmente, trata de reducir el alcance del encuentro para facilitar su labor. Cuando éste adquiere cierto nivel de autonomía, va a disponer de un importante grado de control sobre los clientes, más relevante en el caso de servicios profesionales. Ante esta situación, normalmente, el cliente dispondrá de un mayor nivel de personalización y la empresa verá reducido su nivel de eficiencia.

- Encuentro dominado por el cliente. El dominio por parte del cliente en el encuentro puede tener lugar tanto en servicios estandarizados como en servicios personalizados. En el primer caso es el autoservicio (Bateson, 1985b; Globerson y Maggard, 1991; Dabholkar, 1996a; Meuter y Bitner, 1998; Meuter et al, 2000) el que permite al cliente disponer de la mayoría del control, el resultado del encuentro suele derivar en un alto nivel de eficiencia para la empresa y un elevado grado de satisfacción para el cliente, siempre que éste no necesite ni desee un cierto nivel de servicio por parte del personal en contacto. En el segundo caso puede ser necesaria la utilización de todos los medios por parte de la empresa, lo que supone un elevado coste $y$, por tanto, reduce el nivel de eficiencia.
Por otro lado, los encuentros de servicio pueden configurarse de distintas formas, constituyendo lo que Chase $y$ Aquilano (1998) denominan matriz de diseño servicio-sistema. En esta matriz se utilizan tres dimensiones: grado de contacto entre cliente y servidor, oportunidad de venta y eficiencia de producción.

Cuando el grado de contacto entre el cliente y el servidor -personal en contacto- es nulo, se habla de núcleo aislado por estar físicamente separado del cliente. Se hará referencia a un sistema permeable cuando el cliente puede penetrar a través de contacto telefónico o con su presencia física. Finalmente, cuando el sistema es permeable y reacciona ante los requisitos del cliente, se tratará de un sistema reactivo. La oportunidad de venta, dimensión que podría considerarse una proposición lógica de Marketing, se incrementa a medida que aumenta el grado de contacto, mientras que la eficiencia de producción evoluciona negativamente a medida que el cliente interviene en mayor medida en la operación.

Las distintas entradas de la matriz representan, pues, las diversas maneras en que puede entregarse el servicio. Encontrándose en un extremo el contacto por correo y en el otro el contacto frente a frente y a medida (ej.: servicios médicos), pasando por tecnología en el lugar -in situ(ej.: máquinas expendedoras), contacto por teléfono, contacto frente a frente con especificaciones rígidas (ej.: restaurantes de comida rápida) y el contacto frente a frente con especificaciones flexibles (ej.: restaurantes de servicio completo). 
A medida que el grado de contacto entre cliente y servidor es mayor la eficiencia de producción decrece, pero para contrarrestar esta situación, el contacto frente a frente ofrece una gran oportunidad de venta de productos adicionales. De tal modo, el bajo contacto, como en el caso del correo, permite un mayor grado de eficiencia ya que el cliente no puede afectar de forma importante el sistema, pero en este caso, la oportunidad de venta de productos adicionales será baja. En definitiva, las variables contacto con el cliente y eficiencia productiva están inversamente relacionadas, lo que se explica -al menos en parte- por los elevados precios que habría que fijar para aquellos servicios con altos niveles en las dos variables antes mencionadas.

\subsection{Elementos que intervienen en la prestación del servicio}

Basándose en lo expuesto hasta el momento, puede decirse que para la prestación de un servicio se necesita una serie de factores sintetizados bajo el título de tres elementos -cliente, personal en contacto y organización- cuyas interacciones constituyen lo que se ha dado en llamar Service Encounter.

De este modo, en un primer modelo (véase Figura 1) se distinguen tres elementos: dos personas -elementos de base- y el servicio que es la resultante de la interacción entre los elementos anteriores. Una de las personas será el beneficiario del servicio -cliente- y la otra el prestador del mismo -personal en contacto-, existiendo entre los tres elementos relaciones de carácter recíproco. Un claro ejemplo de este tipo de modelo podría surgir simplemente ante la pregunta que un alumno -beneficiario- hace a un profesor -prestador-, la respuesta sería el servicio prestado.

En un segundo modelo (véase Figura 2), se distinguen también tres elementos, los elementos de base son una persona -el beneficiario, en términos empresariales el cliente- $y$ un bien tangible -soporte físico: elemento perteneciente a la organización-, y el tercer elemento, el servicio, es la resultante de la interacción de los dos primeros. Un ejemplo de este modelo sería aquel que se presenta cuando un cliente obtiene un servicio dirigiéndose a un cajero automático -soporte físico- de una entidad financiera.

\section{Figura 1}

Subsistema de Operaciones en las Empresas de Servicio. Modelo: Prestador -Beneficiario - Servicio

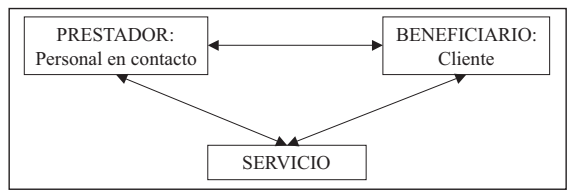

Fuente: Elaboración Propia.

\section{Figura 2}

Subsistema de operaciones

en las empresas de servicio. Modelo: Bien TangibleBeneficiario-Servicio

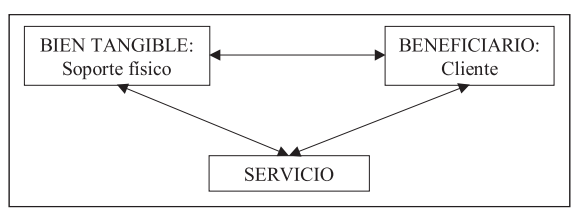

Fuente: Elaboración Propia. 
Con la combinación de los estos dos modelos se obtiene un tercero (véase Figura 3) donde se relacionan cuatro elementos: el servicio, dos personas y un bien tangible. El servicio sigue siendo el output del sistema, y las personas serán el prestador y el beneficiario. Un claro ejemplo surge cuando una paciente -beneficiario- se dirige a su médico -prestador-y éste para atenderle necesita realizarle una radiografía con un aparato de rayos X -soporte físico-, el diagnóstico y el tratamiento serán el servicio prestado.

Una vez tratadas las relaciones entre el cliente, el personal en contacto y la organización -fundamentalmente a través del soporte físico-, se ha considerado apropiado resaltar también aquella parte de la organización no visible a los ojos del cliente pero que marca las pautas de actuación del personal en contacto y el soporte físico, conocida como sistema de organización interna. Por otro lado, también se ha creído conveniente tener en cuenta la presencia de los otros clientes en la prestación del servicio, de hecho autores como Martin y Pranter (1989), Grove y Fisk (1997) reconocen que en muchas ocasiones los consumidores se encuentran físicamente juntos, interactuando con los demás elementos que intervienen en la producción.

De ahí que se haya seguido la propuesta de Eiglier y Langeard (1993: 3-17) a la hora de identificar los elementos del subsistema de operaciones. Estos autores distinguen tres elementos pertenecientes a la empresa -el personal en contacto, el soporte físico y el sistema de organización interna-, otros pertenecientes al mercado -el cliente y los otros clientesy aquellos elementos resultantes de la in- teracción entre los clientes y los elementos de la empresa -los servicios- (véase Figura 4).

Dentro de las relaciones existentes entre los distintos elementos del subsistema de operaciones se han establecido tres grupos:

\section{Figura 3}

Subsistema de Operaciones en las Empresas de Servicios. Modelo: Prestado-Bien tangibleBeneficiario-Servicio

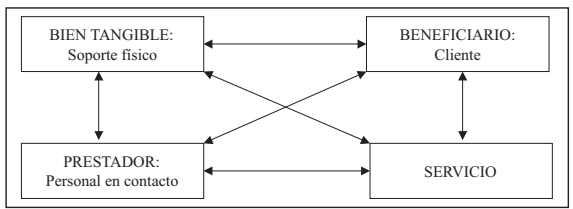

Fuente: Elaboración Propia.

\section{Figura 4}

Subsistema de Operaciones en las empresas de servicio. Elementos que intervienen

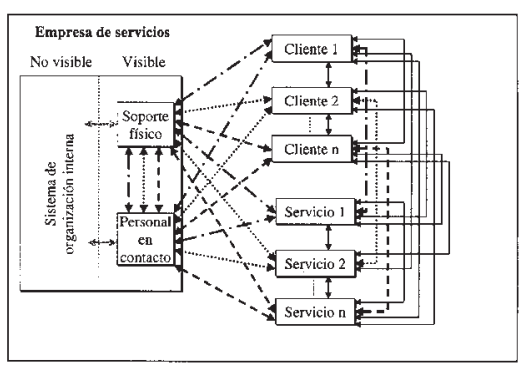

\footnotetext{
-.....- Relaciones primarias de la prestación 1

..... Relaciones primarias de la prestación 2

------ Relaciones primarias de la prestación $n$

_ Relaciones internas

Relaciones de concomitancia
}

Fuente: Elaboración Propia. 
- Relaciones primarias: Son aquellas relaciones básicas necesarias para que la prestación del servicio tenga lugar. No obstante, no en todas las prestaciones están presentes las seis relaciones primarias aquí indicadas, puesto que en ocasiones se podría prescindir del soporte físico o del personal en contacto lo que supondría un número inferior.

- Relaciones internas: Son aquellas que muestran la interacción entre el soporte físico y el personal en contacto con el sistema de organización interna.

- Relaciones de concomitancia: Estas relaciones tienen lugar por la prestación conjunta de distintos servicios, siendo las relaciones existentes entre los clientes, entre los servicios y entre cada cliente y los servicios relativos a los demás clientes. En ciertas empresas se potencia este tipo de relaciones (ej. Pub), mientras que en otras se trata de evitar (ej. consulta médica).

\section{La automatización como posible herramienta}

Tras haber analizado el subsistema de producción de las empresas de servicios, puede decirse que la gestión del mismo debe incluir dos puntos principales: el desarrollo de recursos generadores de servicios y la coordinación de la interacción entre dichos recursos (Grönroos, 1994: 185). Por tanto, la gestión y desarrollo de este subsistema requiere, por un lado, la determinación del volumen de recursos, tanto humanos -personal y clientes- como físicos, que se van a utili- zar. Y por otro, estos recursos deben ser coordinados en un sistema funcional, de tal forma que permitan la transformación del concepto de servicio en servicios que dispongan de la calidad deseada.

Sería difícil conseguir los objetivos de la empresa, tanto si la empresa ha invertido de forma correcta en recursos pero no los gestiona correctamente, como si se realiza una gestión eficiente y eficaz pero los recursos resultan inapropiados o insuficientes. Por tanto, los recursos y su gestión deben adecuarse a los conceptos de servicio definidos con anterioridad, los cuales han de provenir de la misión de la empresa. Por esta razón, las empresas -también las manufactureras- no deben intervenir en la producción de servicios que no se encuentren dentro del ámbito de su misión.

Para poder decidir correctamente sobre los conceptos de servicio y los recursos generadores de los mismos, en la mayoría de los casos, es necesario el estudio del mercado para conocer qué segmentos de clientes potenciales se van a presentar, su importancia, cuáles son sus necesidades y deseos, cuáles son los beneficios que pretenden alcanzar, etc.; de tal forma que una vez interpretados los gustos y necesidades de los clientes potenciales no se cometan errores en la planificación del proceso. Pero además de utilizar la información recogida con la investigación de mercado, deberían tenerse en cuenta las fuentes internas de información, siendo el personal en contacto un importante informador.

Por otro lado, para el desarrollo de este subsistema existen diversas estrategias, el concepto de servicio es el que marcará las líneas maestras a seguir. No obs- 
tante, pueden obtenerse los mismos "resultados" con diversas combinaciones de los recursos generadores del servicio. La estrategia debe adaptarse a los gustos y necesidades de los clientes. Éstos pueden estar capacitados y desear realizar ciertas tareas en el proceso de producción, para los cuales puede resultar interesante establecer ciertos elementos de autoservicio; o bien puede que no sepan o no deseen realizar estas tareas por lo que prefieran que se les atienda personalmente.

En la prestación del servicio -tal como se ha visto en el apartado anteriorno necesariamente deben estar presentes todos los elementos pertenecientes al subsistema de operaciones. Históricamente, todas las relaciones de servicio -en el ámbito comercial- tuvieron lugar con la presencia del consumidor y del personal en contacto. Por ello la investigación académica relativa a la prestación de servicios ha explorado, sobre todo, las características de dichas interacciones (Bitner, Booms y Tetreault, 1990; Lewis y Entwistle, 1990; Rafaeli, 1993; Mohr y Bitner, 1995; Price, Arnould y Deibler, 1995; Bettencourt y Gwinner, 1996; Clemmer y Schneider, 1996; Goodwin, 1996; Goodwin y Gremler, 1996; Hartline y Ferrell, 1996; Fischer et al, 1997 y Gremler et al, 2001). Mucho menos estudiadas están las interacciones del consumidor con los medios tecnológicos (Dabholkar, 1996a; Bitner et al, 2000; Anselmsson, 2001 y Bowden, 2002;). Donde la preocupación fundamental ha sido el desarrollo de perfiles de usuarios (Bateson, 1985b; Zeithaml y Gilly, 1987; Darian, 1987; Greco y Fields; 1991 y Eastlick, 1996), el análisis de las actitudes del consumidor hacia la tecnología (Raub, 1981; Dabholkar, 1992; Dabholkar, 1996a; Parasuraman, 1998; Bobbitt y Dabholkar, 2001; Dabholkar y Bagozzi, 2002; Walter et al, 2002, Dabholkar et al, 2003), y el estudio de la evolución de la satisfacción del cliente (Meuter et al, 2000).

No obstante, la continua proliferación de tecnologías self-service lleva a la necesidad de investigaciones más allá de las dinámicas interpersonales de los service encounters. Diversos investigadores han reconocido la importancia de la tecnología en la ejecución del servicio (Globerson y Maggard, 1991; Fisk et al, 1993; Schneider y Bowen, 1995; Dabholkar, 1996a; Dabholkar, 1996b; Lawrence y Karr, 1996; Parasuraman, 1996; Quinn, 1996; Harvey et al, 1997 y Meuter y Bitner, 1998). Tal es así, que las interacciones en el medio tradicional -marketplaceestán siendo remplazadas por las transacciones en el marketspace (Rayport y Sviokla, 1995), donde las tecnologías self-service se manifiestan como ejemplo clásico de estas últimas.

Ante esta panorámica se ha considerado conveniente estudiar que efectos positivos prodría reportar la automatización sobre los resultados del subsistema de operaciones. Para ello se han tomado como referencia las variables consideradas tradicionalmente objetivos de dicho subistema (Domínguez et al, 1995: 7490): costes, tiempo, calidad, flexibilidad y servicio al cliente.

Respecto al objetivo costes, la incorporación de procesos automatizados podría permitir alcanzar tasas de productividad más elevadas, -ya no sólo por la sustitución de recursos humanos por tecnologías más eficientes sino también por 
el incremento de la participación del cliente-, sin perjudicar o incluso mejorando los resultados relativos a los demás objetivos del subsistema de operaciones.

Por otro lado, a la hora de establecer el tiempo del servicio deben marcarse una serie de retos, entre los que se han destacado los siguientes (Fessard, 1995):

- El cliente debe ser libre y bien servido. El cliente debe ser servido cuando quiera, donde quiera y como quiera. La automatización podría ser una posible vía, sobre todo por lo que se refiere al "cuándo" ya que normalmente permite incrementar la franja horaria de atención al público con mayor facilidad; también respecto al "dónde" porque aunque el personal pueda tener una mayor movilidad, suele resultar menos costoso establecer nuevos puntos de venta a través de la automatización que con mano de obra; ofreciendo más limitaciones para el "cómo" ya que la automatización suele reducir el número de alternativas posibles.

- La empresa deberá ofrecer un servicio libre, permanente e instantáneo. La tendencia es a estar a disposición del cliente la mayor parte del tiempo posible ofreciendo servicios que requieran poco tiempo de preparación, lo que hace pensar en una mayor accesibilidad al servicio y unos menores tiempos de espera, cuestiones ante las que la automatización podría resultar interesante.

- Y, finalmente, el personal exigirá trabajar a tiempo elegido. Teniendo en cuenta que el tiempo del personal en contacto debiera coincidir con el de los clientes, una posible opción podría consistir en sustituir mano de obra por capital.

En relación con el objetivo flexibilidad hay que tener en cuenta que una empresa flexible es aquella que puede manejar la variabilidad perjudicando lo menos posible otras variables como el tiempo, el coste o la calidad. Las fuentes de variabilidad tanto pueden ser internas como externas. Para su correcta gestión habrá que tratar de reducirla y/o incrementar la habilidad para gestionarla en el punto de impacto, si esto es posible.

Siguiendo el modelo propuesto por Harvey et al. (1997) para dirigir la flexibilidad en los servicios, se distinguen cuatro dimensiones relativas a la variabilidad: cambios en volumen, tiempo y lugar, necesidades y clientes. Estos mismos autores utilizan como herramientas para conseguir la flexibilidad deseada: una mayor autonomía de los clientes, la tecnología, los recursos humanos, las modificaciones organizativas y el diseño del proceso y gestión de la red. Por tanto, la automatización podría ser una medida para afrontar la variabilidad externa que no ha sido eliminada en la fuente, sobre todo cuando se trata de cambios en el volumen, en el tiempo y el lugar.

Por otro lado, para mejorar la calidad del servicio hay que tener en cuenta que las características de los mismos proporcionan ciertos inconvenientes (Fernández, 1996). Suelen presentarse ciertas dificultades para medir la calidad directamente relacionada con la intangibilidad, el carácter perecedero y la heterogeneidad. La heterogeneidad, la simultaneidad de la producción y el consumo hacen que tenga lugar una interacción di- 
recta entre el cliente y el productor del servicio, por lo que el personal en contacto tiene un gran protagonismo. Ante esta situación, la incorporación de la automatización parece manifestarse como una vía para incrementar el nivel de calidad, ya que permite ofrecer servicios más homogéneos y con un carácter más tangible, aunque es posible que sea el cliente el que reclame esa heterogeneidad.

Finalmente, por lo que respecta al servicio al cliente, se debe indicar que es el responsable de que en muchas ocasiones no se ofrezca un servicio único sino un conjunto de ellos, siendo de gran importancia la presencia del personal en contacto para el desarrollo del mismo, aspecto que podría perderse con la automatización, aunque está última podría proporcionar otras ventajas como un mayor grado de intimidad o una mayor accesibilidad al servicio.

En definitiva, la automatización podría ser utilizada como una vía para diferenciarse de los demás, proporcionando una mayor accesibilidad, unos menores tiempos de espera, etc.; aunque también podría incorporarse con el objetivo de reducir los costes ${ }^{4}$. Más aún, se ha constatado que con la implantación de sistemas automatizados en la prestación de servicios, pueden lograrse cotas aceptables de productividad y flexibilidad de manera simultánea (Diz y García, 2004), permitiendo, pues, una mayor compatibilidad de dos prioridades competitivas, que tra- dicionalmente eran consideradas como manifiestamente antagónicas.

En la actualidad, la automatización es una auténtica realidad en las empresas del sector terciario, aunque en muchos casos se sigue manteniendo el sistema utilizado antes de la misma con el fin de aprovechar las ventajas de ambos (Humphrey, 1994; Maudos y Pastor, 1995), dando la oportunidad al cliente de elegir qué sistema desea utilizar dependiendo de sus propias preferencias, disponiendo así de un mayor grado de flexibilidad y ofreciendo un mejor servicio al cliente.

\section{Conclusiones}

En el subsistema de operaciones de las empresas de servicios pueden identificarse una serie de elementos: el servicio, el cliente, el personal en contacto, el soporte físico, el sistema de organización interna y los demás clientes. Destacando la importancia del cliente para la existencia de dicho bien, que además de consumidor actúa como coproductor, y la posibilidad de prescindir del personal en contacto para la prestación del servicio. Lo que hace proclive la automatización como una posible alternativa para conseguir los objetivos del subsistema de operaciones.

Entre estos elementos se han establecido una serie de relaciones -primarias, internas y de concomitancia- que medio para poder competir, debido a que las tecnologías utilizadas suelen ser fácilmente imitables, lo que le confiere poca durabilidad a este recurso (Grant, 1991). 
se han visto modificadas al igual que éstos ante la incorporación de procesos automatizados. Estos cambios gestionados correctamente podrían reportar mejoras en los resultados: 1) Se está poniendo de manifiesto una reducción en la presencia del personal en contacto. De tal forma, que incluso en el caso de tareas desarrolladas por intelectuales donde parecía que la automatización no tenía demasiada cabida, la experiencia está mostrando todo lo contrario, 2) Las empresas dejan de ser tan intensivas en mano de obra para ser más intensivas en capital -aunque puede haber casos en los que la intensidad de capital también disminuya- , lo que parece desembocar en una disminución de los costes, 3) Tiene lugar una mayor participación por parte del cliente, lo que también podría reportar mejoras en productividad; y una menor interacción del mismo con el personal en contacto y los demás clientes, permitiendo así un mayor grado de intimidad y 4) La incorporación de procesos automatizados podría permitir una reducción de los tiempos de espera y un incremento del nivel de accesibilidad al servicio, este último provocado por un aumento en el número de puestos de venta -unidades a las que puede acudir el cliente para obtener el servicio, pudiendo estar conformados por personas y/o máquinas- y/o una ampliación del horario de atención al público.

En definitiva, la automatización representa una posible vía para conseguir los objetivos del subsistema de operaciones, siempre teniendo en cuenta que la utilización de esta herramienta no impide el uso de otras, y que no siempre la automatización es la opción más favorable para la empresa, puesto que es importante adaptarse a la situación concreta de cada organización.

\section{Referencias bibliográficas}

Adam, Everett E.y Ebert, Jr. Ronald (1991), Administración de la producción y las operaciones, Prentice-Hall. México.

Agnihothri, Saligrama; Sivasubramaniam, Nagaraj y Simmons, Donald (2002), Levering technology to improve field service, International Journal of Service Industry Management, Vol. 13, No. 1, pp. 47-68. Inglaterra.

Anselmsson, Johan (2001), Customer-perceived service quality and technology-based self-service, doctoral dissertation, Lund, Lund University, Lund Business Press. Suecia.

Bateson, John E. (1985a), Perceived Control and the Service Encounter, en Czpiel, John A.; Solomon, Michael R. y Surprenant, Carol F. (Eds.), The Service Encounter, MA, Lexington, Lexington Books. USA.

Bateson, John E. (1985b), Sef Service Consumer: An Exploratury Study, Journal of Retailing, Vol. 61, No. 3, pp. 49-76. New York. USA.

Bettencourt, Lance y Gwinner, Kevin (1996), Customization of the Service Experience: The Role of the Frontline Employee, International Journal of Service Industry Management, Vol. 7, No. 2, pp. 2-20. Inglaterra.

Bitner, Mary Jo; Booms, B.H. y Tetreault, M.S. (1990), The Service Encounter: Diagnosing Favorable and Unfavorable Incidents, Journal of Marketing, Vol. 54, Janaury, pp. 71-84. USA.

Bitner, Mary Jo; Brown, Stephen W. y Meuter, Matthew L. (2000), Technology Infusion in Service Encounters, Journal 
of the Academy of Marketing Science, Vol. 28, No. 1, pp. 138-149. Miami. USA.

Bowden, Bobby (2002), Customers help check out new technology, Arkansas Business, Vol. 19, No. 5, pp. 15-18. USA.

Chase, Richard B. (1988), Service System Productivity Requires More Than a Stopwatch, Working Paper, University of Southern California Graduate Sclool of Business Administration. USA.

Chase, Richard B.; Aquilano, Nicholas J. y Jacobs, F. Robert (1998), Production and Operations Management: Manufacturing and Services, Irwin McGraw-Hill. Boston. USA.

Clemmer, Elizabeth C. y Schneider, Benjamin (1996), Fair Service, en Swartz, Teresa A.; Bowen, David E. y Brown, Stephen W. (Eds.), Advances in Services Marketing and Management, CT, Greenwich, JAI Press, Vol. 5, pp. 109-126. Inglaterra.

Dabholkar, Pratibha A. (1992), Role of Affect and Need for Interaction in On-Site Service Encounters, en Sherry, J.F. and Sternthal, B. (Eds.), Advances in Consumer Research, UT, Provo, Association for Consumer Research, Vol. 19, pp. 563-569. USA.

Dabholkar, Pratibha A. (1996a), Consumer Evaluations of New Technology-Based Self-Service Options: An Investigation of Alternative Models of Service Quality, International Journal of Research in Marketing, Vol. 13, No 1, pp. 29-51. USA.

Dabholkar, Pratibha A. (1996b), TechnologyBased Service Delivery: A Classification Scheme for Developing Marketing Strategies, en Swartz, T.A., Bowen, D.E. and Brown, S.W. (Eds.), Advances in Services Marketing and Management, CT, Greenwich,
JAI Press, Vol. 3, pp. 241-271. Inglaterra.

Dabholkar, Pratibha A. y Bagozzi, Richard P. (2002), An attitudianl model of technology-based sef-service: moderating effects of consumer traits and situational factors, Journal of the Academy of Marketing Science, Vol. 30, No. 3, pp. 184-201. Miami. USA.

Dabholkar, Pratibha A.; Bobbitt, L. Michelle y Lee, Eun-Ju (2003), Understanding consumer motivation and behavior related to self-scanning in retailing, International Journal of Service Industry Management, Vol.14, No. 1, pp. 59-95. Inglaterra.

Darian, J.C. (1987), In-Home Shopping: Are There Consumer Segments?, Journal of Retailing, Vol. 63, No. 2, pp. 163-186. New York. USA

Diz Comesaña, María Eva y García Vázquez, José Manuel (2004), Impacto de la automatización en las empresas de servicios: Un estudio empírico, actas de las XIV Jorandas Luso Espanholas de Gestão Científica. 4 al 7 de Febrero, Universidade dos Açores. Ponta Delgada. Portugal.

Domínguez Machuca, José Antonio; Álvarez Gil, María José; Domínguez Machuca, M.Ángel; García González, Santiago; Ruiz Jiménez, Antonio (1995), Dirección de Operaciones. Aspectos estratégicos en la producción y los servicios, McGraw-Hill. Madrid.

Eastlick, M.A. (1996), Consumer Intention to Adopt Interactive Teleshopping, Marketing Science Institute Working Paper, MA, Cambridge, Marketing Science Institute, pp. 96-113. United Kingdom

Eiglier, Pierre y Langeard, Eric (1993), Servucción. El Marketing de Servicios, McGraw-Hill. Madrid.

Fernández Barcala, Marta (1996), Cómo medir la Calidad de los Servicios, ICE Tribu- 
na de Economía, No. 755, July, pp.113-125. Madrid.

Fessard, Jean-Luc (1995), El tiempo del servicio. El reto del tiempo en las actividades de servicio, Marcombo Boixareu Editores. Barcelona. España.

Fischer, Eileen; Gainer, Brenda y Bristor, Julia (1997), The Sex of the Service Provider: Does it Influence Perceptions of Service Quality?, Journal of Retailing, Vol. 73, No. 3, pp. 361-382. New York. USA.

Fisk, Raymond P.; Brown, Stephen W. y Bitner, Mary Jo (1993), Tracking the Evolution of the Services Marketing Literature, Journal of Retailing, Vol. 69, No. 1, pp. 61-103. New York. USA.

Globerson, Shlomo y Maggard, Michael J. (1991), A Conceptual Model of Selfservice, International Journal of Operations and Production Management, Vol. 11, No. 4, pp. 33-43. Ohio. USA.

Goodwin, Cathy (1996), Communality as a Dimension of Service Relationships, Journal of Consumer Psychology, Vol. 5, No. 4, pp. 387-415.

Goodwin, Cathy y Gremler, Dwayne D. (1996), Friendship Over the Counter: How Social Aspects of Service Encounters Influence Consumer Service Loyalty, en Swartz, T.A., Bowen, David E. y Brown, Stephen W. (Eds.), Advances in Services Marketing and Management, CT, Greenwich, JAI Press, Vol. 5, pp. 247-82. Inglaterra.

Grant, R.M. (1991), The Resource-based Thory of Competitive Advanytage: Implications for Strategy Formulation, California Management Review, Vol. 33, No. 3, pp. 114-135. USA.

Greco, Alan J. y Fields, D.Michael (1991), Profiling Early Triers of Service Innovations: A Look at Interaction Home Video Ordering Services, Journal of
Services Marketing, Vol. 5 No. 3, pp. 19-26.

Gremler, Dwayne D.; Gwinner, Kevin P. y Brown, Stephen W. (2001), Generating positive word-of- mouth communication through customer-employee relationships, International Journal of Service Industry Management, Vol. 12, No 1, pp. 44-59. Inglaterra.

Grönroos, Cristian (1994), Marketing y gestión de servicios. La gestión de los momentos de la verdad y la competencia de los servicios, Díaz de Santos. Madrid

Grove, Stephen J. y Fisk, Raymond P. (1997), The impact of Other Customers on Service Experiences: A Critical Incident Examination of 'Getting Along', Journal of Retailing, Vol. 73, No. 1, pp. 217-224. New York. USA

Hartline, Michael D. y Ferrell, O.C. (1996), The Management of Customer-Contact Service Employees: An Empirical Investigation, Journal of Marketing, Vol. 60, October, pp. 52-70. USA.

Harvey, Jean; Lefebvre, Louis A. y Lefebvre, Elisabeth (1997), Flexibility and technology in services: a conceptual model, International Journal Operations \& Production Management, Vol. 17, No. 1, pp. 29-45. Ohio. USA.

Humphrey, David B. (1994), Delivering Deposit Services: ATMs Versus Branches, Economic Quarterly, vol. 80, spring, pp. 59-81. USA.

Lawrence, P. y Karr, John (1996), Technology Spending and Alliances: New Highs in Financial Services Firms, Journal of Retail Banking Services, Vol. 17, No 3, pp. 45-52. USA.

Lewis, Barbara R. y Entwistle, Thomas W. (1990), Managing the service encounter: a focus on the employee, International Journal of Service Industry Management, vol. 1, No. 3, pp. 41-52. Inglaterra. 
Martin, Charles L. y Pranter, Charles A. (1989), Compatibility Management: Customer-to-Customer Relationships in Service Environments, Journal of Services Marketing, Vol. 3, Summer, pp. 6-15.

Maudos Vilarrolla, Joaquín y Pastor, José Manuel (1995), Economías de gama en la prestación de servicios bancarios: Cajeros automáticos versus oficinas, actas de la IX Reunión ASEPELT. 22 y 23 de Junio, Vol. IV, pp. 81-90. Santiago de Compostela. España.

Meuter, Matthew L. y Bitner, Mary Jo (1998), Self-Service Technologies: Extending Service Frameworks and Identifying Issues for Research, en Dhruv Grewal y Connie Pechmann (Eds.), AMA Winter Educators' Conference Proceedings: Marketing Theory and Applications, American Marketing Association, Vol. 9, pp. 12-19. USA.

Meuter, Matthew L.; Ostrom, Amy L.; Roundtree, Robert I. y Bitner, Mary Jo (2000), Self-Service Technologies: Understanding Customer Satisfaction with Technology-Based Service Encounters, Journal of Marketing, Vol. 64, July, pp. 50-64. USA.

Mohr, Lois A. y Bitner, Mary Jo (1995), Process Factors in Service Delivery: What Employee Effort Means to Customers, en Swartz, T.A., Bowen, D.E. y Brown, S.W. (Eds.), Advances in Services Marketing and Management, CT, Greenwich, JAI Press, Vol. 4, pp. 91117. Inglaterra.

Mulligan, Paul y Gordon, Steven R. (2002), The impact of information technology on customer and supplier relationships in the financial services, International Journal of Service Industry Management, Vol. 13, No. 1, pp. 29-46. Inglaterra.

Normann, Richard (1990), La gestión en las empresas de servicios. Estrategia y liderazgo, Deusto. Bilbao. España.
Parasuraman, A. (1996), Understanding and Leveraging the Role of Customer Service in External, Interactive and Internal Marketing, paper presented at Frontiers in Services Conference, TN, Nahville, October. USA.

Parasuraman, A. (1998), Customers' Confort Level with Technology-Based Services: An empirical Study, paper presented at AMA winter Educators' Conference, TX, Auxtin, February 21-24. USA.

Price, Lidda L.; Arnould, Eric J. y Deibler, Sheila L. (1995), Consumers' Emotional Responses to Service Encounters, International Journal of Service Industry Management, Vol. 6, No. 3, pp. 34-63. Inglaterra

Quinn, James Brian (1996), The Productivity Paradox Is False: Information technology Improves Service Performance, en Swartz, T.A., Bowen, D.E. y Brown, S.W. (Eds.), Advances in Services Marketing and Management, CT, Greenwich, JAI Press, Vol. 5, pp. 7184. Inglaterra.

Raub, A.C. (1981), Correlates of Computer Enxiety in College Students, doctoral dissertation, Universsity of Pennsylvania, Chemistry Department. USA.

Rafaeli, Anat (1993), Dress and Behavoir of Customer Contact Employees: A Framework for Analysis, en Swartz, T.A., Bowen, D.E. y Brown, S.W. (Eds.), Advances in Services Marketing and Management, CT, Greenwich, JAI Press, Vol. 4, pp. 175-211. Inglaterra

Rayport, Jeffrey F. y Sviokla, John J. (1995), Exploiting the Virtual Value Chain, Harvard Business Review, Vol. 73, November-December, pp. 14-24. USA.

Schlessinger, Leonard A. y Heskett, James L. (1991), The Service-Driven Service 
Company, Harvard Business Review, September-October. USA.

Schneider, Benjamin y Bowen, David E. (1995), Winning The Service Game, Boston, Harvard Business School Press. USA.

Shostack, G. Lynn (1984), Designing Services That Deliver, Harvard Business Review, Enero-Febrero. USA.

Shostack, G. Lynn (1987), Service Positioning Through Structural Change, Journal of Marketing, Enero. USA.

Solomon, Michael R.; Surprenant, Carol; Czpiel, John A. y Gutman, Evelyn G. (1985), A role theory perspective on dynamic interactions: the service encounter, Journal of Marketing, Vol. 49, pp. 99-111. USA.
Tansik, David A. (1988), Balance in Service Systems Desibn, Informe de investigación presentado en el Seinsheimer Symposium on Business, Freemen School of Business, Tulane University, 12 noviembre. New Orleans. USA.

Walker, Rhett H.; Craig-Lees, Margaret; Hecker, Robert y Francis, Heather (2002), Technology-enabled service delivery: an investigation of reasons affecting customer adoption and rejection, International Journal of Service Industry Management, Vol. 13, No. 1, pp. 91-110. Inglaterra.

Zeithaml, Valarie y Gilly, Mary C. (1987), Characteristics Affecting the Acceptance of Retailing Technologies: A Comparison of Elderly and Nonelderly Consumers, Journal of Retailing, Vol. 63, No. 1, pp. 49-68. New York. USA. 